\title{
Role of Hyperbaric Oxygen Therapy in Traumatic Brain Injury-Evidence-Based Consensus
}

\author{
Vernon Velho ${ }^{1}$ Hrushikesh Kharosekar ${ }^{1} \quad$ Laxmikant Bhople $^{1} \quad$ Deepak A. Palande ${ }^{1}$ \\ ${ }^{1}$ Department of Neurosurgery, Sir J J Group of Hospitals and Grant \\ Medical College, Mumbai, Maharashtra, India

\begin{abstract}
Address for correspondence Hrushikesh U. Kharosekar, MCh, Department of Neurosurgery, 4th Floor, Grant Medical College and Sir J J Group of Hospitals, Byculla E, Mumbai 400008, Maharashtra, India (e-mail: hkharosekar@gmail.com).
\end{abstract}

Indian J Neurotrauma:2020;17:42-45

\begin{abstract}
Keywords

- hyperbaric oxygen therapy

- traumatic brain injury

Traumatic brain injury (TBI) is a major health problem in India and worldwide, resulting in significant morbidity, mortality, and disabilities of young and productive group of society. Various treatment modalities in the immediate period following a TBI are focused on altering the acute pathophysiology. Secondary injury is precipitated by ischemia resulting from decreased cerebral blood flow leading to hypoxia and is particularly likely to occur in the first 24 hours after injury. Hyperbaric oxygen therapy (HBOT) targets TBI-induced ischemia by exposing patients to an environment that substantially increases the amount of $\mathrm{O}_{2}$ inspiration $\left(100 \% \mathrm{O}_{2}\right.$ at $>1$ atmosphere absolute), producing an increased $\mathrm{O}_{2}$ concentration in the plasma and thus increased delivery of $\mathrm{O}_{2}$ for diffusion to brain tissue. Despite the capacity of HBOT to protect against secondary ischemic damage, the use of HBOT for the treatment of TBI has been controversial. To gain acceptance in routine clinical use, a clinical method of assessing its effectiveness in the individual patient, computed tomography perfusion scan, single-photon emission computed tomography scans, and other intermediate indicators of the effects of HBOT should be examined by large and high-quality studies.
\end{abstract}

\section{Introduction}

Traumatic brain injury (TBI) is a major health problem in India and worldwide, resulting in significant morbidity, mortality, and disabilities of young and productive group of society. It has enormous social and economic impacts across a large variety of populations. As most of the patients suffering from TBI are young adults, saving their lives and improving the outcome remain the goal. Despite the physical and financial impact on individual and society, there has been little advancement in the acute treatment of TBI in past 50 years, and clinical outcomes have not improved much. ${ }^{1}$

Various treatment modalities in the immediate period following a TBI are focused on altering the acute pathophysiology. The most common pathological mechanism in primary injury is diffuse shearing of axonal pathways and small blood vessels, also known as diffuse axonal injury. However, following the primary mechanical injury to the brain, secondary injury frequently develops. This secondary injury is precipitated by ischemia resulting from decreased cerebral blood flow (CBF) leading to hypoxia and is particularly likely to occur in the first 24 hours after injury. Because of decreased oxygen $\left(\mathrm{O}_{2}\right)$ delivery to brain cells, the brain converts from aerobic to highly inefficient anaerobic metabolism, resulting in inadequate energy production in the brain and eventual cell death. Due to the diffuse nature of injury, affecting multiple brain areas, cognitive impairments are usually the predominant symptoms. Global brain hypoperfusion, and its related tissue ischemia, detected in patients suffering from TBI, serves as a rate-limiting factor for any regenerative process. ${ }^{1-3}$

Hyperbaric oxygen therapy (HBOT) targets TBI-induced ischemia by exposing patients to an environment that substantially increases the amount of $\mathrm{O}_{2}$ inspiration $\left(100 \% \mathrm{O}_{2}\right.$ at $>1$ atmosphere absolute [ATA]), producing an increased $\mathrm{O}_{2}$ concentration in the plasma and thus increased delivery of $\mathrm{O}_{2}$ for diffusion to brain tissue. By increasing the oxygen level in blood and body tissues, HBOT can augment the repair
DOI https://doi.org/

10.1055/s-0040-1713312

ISSN 0973-0508.
License terms

() (1) $\odot \circledast$ 
mechanisms. Various models have strongly suggested that HBOT can induce angiogenesis, improve brain plasticity, enhance neurogenesis and synaptogenesis, and foster functional recovery.

Despite the capacity of HBOT to protect against secondary ischemic damage, the use of HBOT for the treatment of TBI has been controversial. One concern regarding the use of HBOT for acute TBI arises from apparent conflicts in the literature regarding its efficacy. It is likely that injury heterogeneity, variable injury chronicity, and variability in study design have contributed to this perception. Additional concerns relate to $\mathrm{O}_{2}$ toxicity and the logistics of widespread implementation of this therapy. ${ }^{1,2}$

\section{History}

HBOT is defined by Undersea \& Hyperbaric Medical Society as a treatment in which a patient intermittently breathes $100 \%$ oxygen while the treatment chamber is pressurized to a pressure greater than sea level (1 ATA). The pressure increase must be systemic, and may be applied in monoplace or multiplace chambers.

The history of hyperbaric medicine reaches back to the year 1620 when Drebbel developed a one-atmosphere diving bell, and 40 years later Boyle joined forces with Gay-Lussac to develop the General Gas Law. Moving the sands of time to the near present day, the modern age of hyperbaric medicine began in 1937, when Behnke and Shaw used a hyperbaric chamber to treat decompression sickness (DCS). However, it was not until 1955 that there was major interest in using hyperbaric oxygenation outside of treating DCS. That year, Churchill-Davidson began to use oxygen therapy in a hyperbaric chamber to treat the damage induced by radiotherapy in cancer patients.

But it was until 1992 when Rockswold (USA) conducted first double-blind randomized controlled trial showing that HBOT reduces mortality in acute TBI by $59 \%$, the largest single reduction in mortality since the invention of the ambulance. In 2002, US Army study confirms Harch's HBOT repairs white matter damage in children with cerebral palsy (CP) and a Canadian group shows hyperbaric air (the original treatment for DCS and Mountain Sickness) and HBOT 1.75 effective in treating $\mathrm{CP}$ in double-blind randomized controlled trial.

Many peer-reviewed articles have been published in the last decade that demonstrate HBOT is effective at repairing an injured brain even long after that injury took place. One of the most notable was the article published by Harch et al using 40 sessions of 60 -minute treatments at 1.5 atmospheric pressure. The blast-induced TBI war veterans experienced a 15 -point IQ increase $(p<0.001), 39 \%$ reduction in postconcussion symptoms, $30 \%$ reduction in post-traumatic stress disorder symptoms, and $51 \%$ decrease in depression. This is all consistent with past-published reports of HBOT in chronic brain injury. The first battle casualty to be treated with HBOT (1.5), was of General Patt Maney (retired) for his blast-induced brain injury in Afghanistan. His treatment was ordered after 9 months of therapy at Walter Reed had shown minimal improvement. As a result of his injuries, he was nonfunctional and unable to return to his job. After HBOT treatment, he was discharged from Walter Reed and returned to his civilian job as a Florida state judge. ${ }^{2}$

\section{Review of Literature}

PubMed search for clinical studies on use of HBOT in TBI was done. There are few clinical studies in literature where HBOT was used in TBI. Though results in these studies were encouraging and showed significant improvement in patients, absence of standard test to analyze results was limiting factor.

\section{Our Study}

A prospective study was conducted in our Department of Neurosurgery, Grant Medical College \& Sir J J Group of Hospitals, Mumbai, India, from August 2006 till August 2019. A total of 1,840 patients with TBI were analyzed in this study. Out of these, 1,260 patients with moderate to severe brain injuries were subjected to HBOT after stabilization or operative intervention and weaning from ventilator. Patients with hemodynamic instability and associated chest injuries were excluded and were included in control group $(n=560)$. Among the patient analyzed, more than $80 \%$ were male patients in both groups (-Table 1). Distribution of type of head injury on computed tomography (CT) scan in both groups is shown in - Table 2. Our results showed that more than 30\% patients showed rapid improvement in Glasgow Coma Scale (GCS) score as compared with only $10 \%$ in control group (-Tables 3 and $\mathbf{4}$, - Fig. 1). Also, there was more than 50\% reduction in hospital stay in study group compared with control group when GCS was more than 9 at admission

Table 1 Demographic distribution

\begin{tabular}{|l|l|l|}
\hline Sex group & $\begin{array}{l}\text { HBOT group } \\
\text { TBI } \\
(n=1280)\end{array}$ & $\begin{array}{l}\text { Control group } \\
(n=560)\end{array}$ \\
\hline Male & $1008(78.9 \%)$ & $440(79.7 \%)$ \\
\hline Female & $272(21.1 \%)$ & $120(20.3 \%)$ \\
\hline
\end{tabular}

Abbreviations: HBOT, hyperbaric oxygen therapy; TBI, traumatic brain injury.

Table 2 Types of traumatic brain injury in both groups

\begin{tabular}{|l|l|l|}
\hline Type of TBI & $\begin{array}{l}\text { HBOT group } \\
(\boldsymbol{n}=1280)\end{array}$ & $\begin{array}{l}\text { Control group } \\
(\boldsymbol{n}=560)\end{array}$ \\
\hline $\begin{array}{l}\text { Extra axial } \\
\text { hematoma }\end{array}$ & $448(35.0 \%)$ & $128(23 \%)$ \\
\hline SDH \pm contusion & $383(29.9 \%)$ & $129(23.2 \%)$ \\
\hline SAH & $155(12.1 \%)$ & $98(17.4 \%)$ \\
\hline $\begin{array}{l}\text { Diffuse axonal } \\
\text { injury }\end{array}$ & $253(19.7 \%)$ & $114(20.3 \%)$ \\
\hline Pure contusion & $41(3.18 \%)$ & $91(16.25 \%)$ \\
\hline
\end{tabular}

Abbreviations: HBOT, hyperbaric oxygen therapy; SAH, subarachnoid hemorrhage; SDH, subdural hematoma; TBI, traumatic brain injury. 
Table 3 GCS score in HBOT and control group on admission

\begin{tabular}{|l|l|l|}
\hline GCS & $\begin{array}{l}\text { HBOT group } \\
\text { (traumatic) } \\
(\boldsymbol{n}=1280)\end{array}$ & $\begin{array}{l}\text { Control group } \\
(\boldsymbol{n}=560)\end{array}$ \\
\hline $13-15$ & $449(35.1 \%)$ & $196(35.0 \%)$ \\
\hline $9-12$ & $669(52.2 \%)$ & $290(51.7 \%)$ \\
\hline$\leq 8$ & $162(12.7 \%)$ & $74(13.3 \%)$ \\
\hline
\end{tabular}

Abbreviations: GCS, Glasgow Coma Scale; HBOT, hyperbaric oxygen therapy.

Table 4 Change in GCS at discharge (after 10 cycles HBOT)

\begin{tabular}{|l|l|l|}
\hline GCS & $\begin{array}{l}\text { HBOT group } \\
\text { (traumatic) } \\
(n=1280)\end{array}$ & $\begin{array}{l}\text { Control group } \\
(n=560)\end{array}$ \\
\hline+3 & $99(7.8 \%)$ & $19(3.5 \%)$ \\
\hline+2 & $268(21.0 \%)$ & $59(10.5 \%)$ \\
\hline+1 & $791(61.8 \%)$ & $341(60.8 \%)$ \\
\hline 0 & $122(9.6 \%)$ & $141(25.2 \%)$ \\
\hline
\end{tabular}

Abbreviations: GCS, Glasgow Coma Scale; HBOT, hyperbaric oxygen therapy.
( - Table 5, - Fig. 2). We have analyzed the results using GCS score, functional outcome, and CT perfusion scan, but still tools for analyzing the benefits of HBOT are very limited.

\section{Discussion}

\section{Mechanism of HBOT}

During the acute phase of a severe TBI, the metabolic demands of the brain increase but $\mathrm{O}_{2}$ delivery to the brain decreases due to a reduction in $\mathrm{CBF}$ as well as barriers to $\mathrm{O}_{2}$ diffusion are caused by capillary endothelial edema, which is exacerbated by the neuroinflammatory response to trauma, capillary collapse, and increased intracranial pressure (ICP). This $\mathrm{O}_{2}$ deficiency forces a conversion to anaerobic metabolism, leading to the depletion of cellular energy (adenosine triphosphate, ATP) and eventually to cell death. The cellular energy crisis resulting from inadequate $\mathrm{O}_{2}$ delivery results in electrolyte imbalances, stemming from the lack of energy for normal $\mathrm{Na}^{+} / \mathrm{K}+$ ATP-ase pump function within neurons and glial cells. This imbalance leads to an increased calcium influx, resulting in an abnormally elevated release of excitatory neurotransmitters and further disruption of mitochondrial metabolism in a positive feed-forward manner that causes excessive free-radical buildup. As the

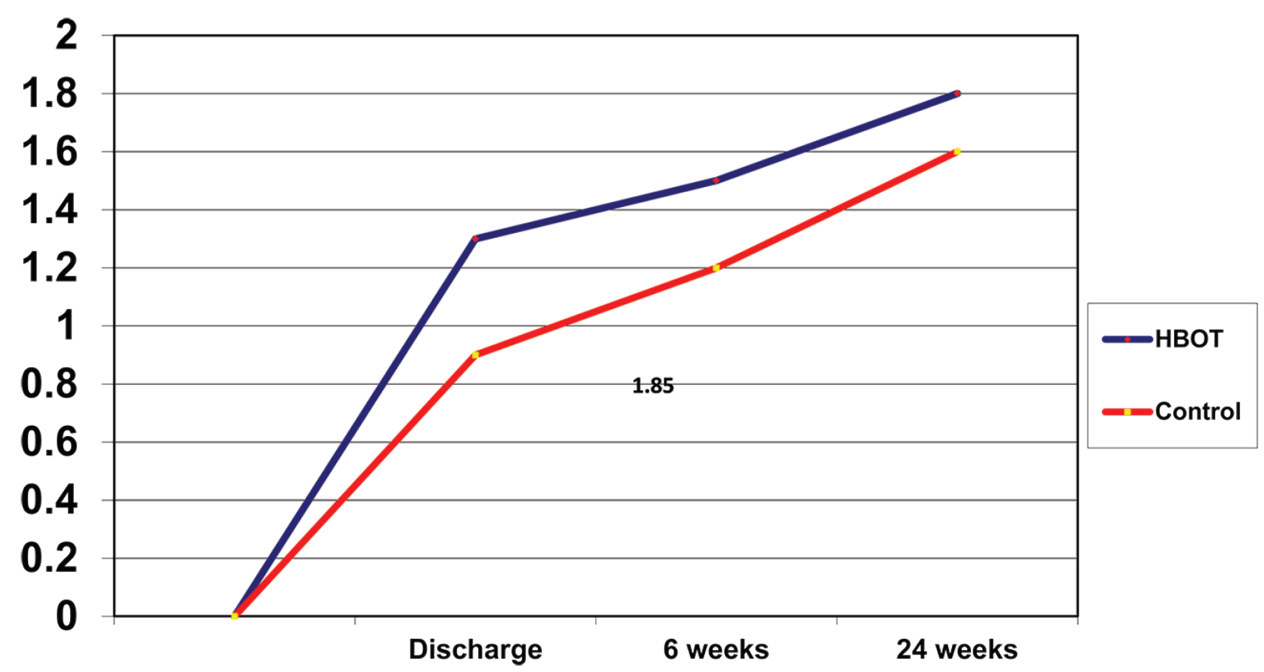

Fig. 1 Functional outcome chart. HBOT, hyperbaric oxygen therapy.

Table 5 Duration of hospital stay

\begin{tabular}{|l|l|l|}
\hline GCS & $\begin{array}{l}\text { HBOT group } \\
n=1280\end{array}$ & $\begin{array}{l}\text { Control group } \\
n=560\end{array}$ \\
\hline $13-15$ & $5-18 d$ (mean: 7.4$)$ & 5-20 d (mean: 12.2$)$ \\
\hline $9-12$ & 7-25 d (mean: 11.4$)$ & $9-30 d$ (mean: 18.5$)$ \\
\hline$<8$ & $14-36 d$ (mean: 21.5$)$ & 15-40 d (mean: 25.5) \\
\hline
\end{tabular}

Abbreviations: GCS, Glasgow Coma Scale; HBOT, hyperbaric oxygen therapy.

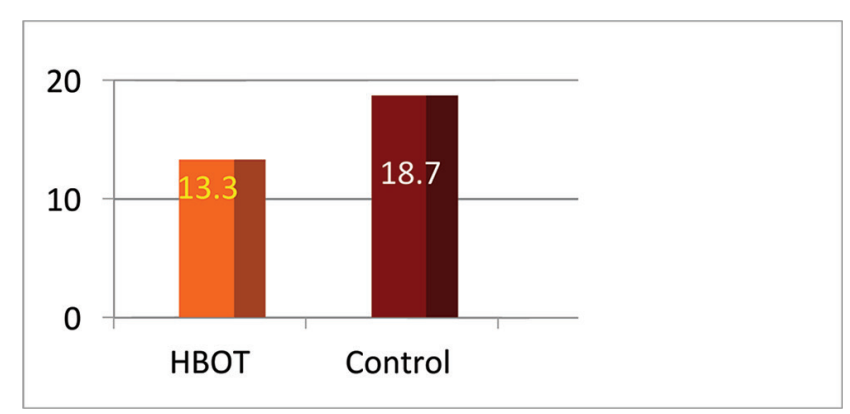

Fig. 2 Histographic presentation of duration of hospital stay. 
neuroinflammatory response continues, apoptosis-mediator proteins such as bcl-2 and bcl-xL initiate the process of cell death. This biochemical cascade resulting in potentially large-scale cell death demonstrates the need for providing an adequate $\mathrm{O}_{2}$ supply following TBI to limit secondary ischemic injury. The effects of HBOT are mediated by increasing the $\mathrm{O}_{2}$ dissolved in plasma, as opposed to the $\mathrm{O}_{2}$ carried by hemoglobin. For example, the dissolved $\mathrm{O}_{2}$ content (volume \%) at room air (1 ATA) is 0.32 . At 1.5 ATA, it is increased by a factor of 10.42 . When additional $\mathrm{O}_{2}$ becomes available for diffusion across capillary endothelium, anaerobic metabolism converts back to aerobic metabolism, allowing mitochondria to restore depleted cellular energy. This neuroprotective effect can be objectively observed in the traumatized human brain by improved cerebral metabolic rate of oxygen $\left(\mathrm{CMRO}_{2}\right)$ measurements following HBOT treatments, as mitochondrial metabolism accounts for $>90 \%$ of $\mathrm{O}_{2}$ consumption in the brain. This neuroprotective increase in $\mathrm{CMRO}_{2}$ leads to several physiologic benefits. First, returning to aerobic metabolism results in improved energy production and halts the cascade toward cell death described above. Second, the averted energy crisis allows for a return of normal autoregulation, which can normalize CBF and ICP. Third, it decreases the neuroinflammatory response that leads to apoptosis. Fourth, as ATP becomes available from restored mitochondrial function, the function of $\mathrm{Na}^{+} / \mathrm{K}+$ ATP-ase pumps improves, allowing osmotic effects to alleviate endothelial swelling and edema. In turn, this reverse induced barriers to the diffusion of $\mathrm{O}_{2}$ to the mitochondria. Finally, the bloodbrain barrier stabilizes and there are increases in stem cell production. HBOT has also been proposed as a treatment for the chronic sequelae of TBI, but evidence to support HBOT for this purpose is weak. Previous review articles have suggested that issues with methodology and statistical analysis may be underlying reasons, but the biochemical mechanism responsible for the benefits of HBOT in chronic mild TBI is not well documented in either clinical or preclinical work. ${ }^{2,3}$

\section{Controversy Surrounding the Use of HBOT}

The biochemical mechanisms and physiologic benefits of acutely administered HBOT for severe TBI provide objective evidence supporting the use of this treatment in the clinical setting. However, controversy still exists due to safety concerns of an increased $\mathrm{O}_{2}$ dose, how meaningful the benefits in functional outcome are, the feasibility of implementing these treatments, and the apparent inability to consistently replicate data.

\section{Safety}

One safety concern related to the therapeutic use of HBOT in TBI stems from $\mathrm{O}_{2}$ toxicity, which is caused by oxidative stress and the formation of reactive $\mathrm{O}_{2}$ species in the lungs and brain tissue after prolonged exposure to $\mathrm{O}_{2}$. Oxygen toxicity is commonly measured in increments of unit pulmonary toxicity dose (UPTD). A treatment consisting of 60 minutes of HBOT at 1.5 ATA with compression/decompression at 2 feet/minute generates 130 UPTD. At a pressure of 2.5 ATA, using the same procedure, the $\mathrm{O}_{2}$ dose is 296 UPTD. Both paradigms are well below the accepted upper limit. It is important to note that interruptions in $\mathrm{O}_{2}$ exposure between treatments have been shown to increase $\mathrm{O}_{2}$ tolerance and improve safety; for example, 600 UPTD per day in two treatment sessions was administered for weeks without any evidence of accumulative pulmonary toxicity. ${ }^{2}$

\section{Feasibility}

Questions have been raised regarding the feasibility of HBOT, because its use requires hospitals to purchase chambers. However, a higher-cost, multiple-occupancy, large compartment chamber requiring sophisticated operation is not necessary for most hospitals. A lower-cost monoplace chamber, which allows for the treatment of a single patient with external support, is entirely adequate and can be incorporated into a critical care area. Given the widespread demographic that TBI affects, the widescale implementation of an effective treatment option for these severely injured patients should be seen as an investment rather than a cost. ${ }^{2}$

\section{Mixed Results}

A major concern of implementing HBOT as a clinical treatment arises from the perception that the data are not consistently replicated in the literature; main factors may contribute to these inconsistencies. The first factor is the heterogeneous pathophysiology of TBI. HBOT may not be the best choice for all patients who present with a severe TBI.,3

\section{Conclusion}

This systematic and comprehensive literature review demonstrates that, despite the controversy surrounding HBOT for the treatment of TBI, this therapy has significant clinical potential. To gain acceptance in routine clinical use, a clinical method of assessing its effectiveness in the individual patient, CT perfusion scan, single-photon emission computed tomography scans, and other intermediate indicators of the effects of HBOT should be examined by large and high-quality studies. A longitudinal cohort study in which all patients undergo proper diagnostic evaluation as well as standardized follow-up tests is necessary.

\section{Conflict of Interest}

None.

\section{References}

1 Hadanny A, Abbott S, Suzin G, Bechor Y, Efrati S. Effect of hyperbaric oxygen therapy on chronic neurocognitive deficits of post-traumatic brain injury patients: retrospective analysis. BMJ Open 2018;8(9):e02338710.1136/ bmjopen-2018-023387

2 Stoller KP. . All the right moves: the need for the timely use of hyperbaric oxygen therapy for treating TBI/CTE/PTSD All the right moves: the need for the timely use of hyperbaric oxygen therapy for treating TBI/CTE/PTSD. Med Gas Res 2015;5:710.1186/s13618-015-0028-0

3 Hu Q, Manaenko A, Xu T, Guo Z, Tang J, Zhang JH. Hyperbaric oxygen therapy for traumatic brain injury: bench-to-bedside. Med Gas Res 2016;6(2):102-110 